\footnotetext{
${ }^{2}$ M. A. Locchi and P. Picchi, Nuovo Cimento 57, 803 (1968); A. Reitan, Nucl. Phys. B68, 387 (1974).

${ }^{3}$ W. R. Gibbs, Phys. Rev. C $\underline{3}, 1127$ (1971), and $\underline{5}$, 775 (1972).

${ }^{4}$ W. R. Gibbs, J. C. Jackson, and W. B. Kaufmann, Phys. Rev. C 9 , 1340 (1974).

${ }^{5}$ L. L. Foldy and J. D. Walecka, Ann. Phys. (N.Y.) 54, 447 (1969); R. H. Landau and F. Tabakin, Phys. Rev. D $\underline{5}, 2746$ (1972); W. R. Gibbs, Phys. Rev. C 10 , 2166 (1974); J. T. Londergan, K. W. McVoy, and E. J. Moniz, Ann. Phys. (N.Y.) 86, 147 (1974).

${ }^{6}$ Y. Shamai et al., preceding Letter [Phys. Rev. Lett. 36,82 (1976)].

${ }^{7}$ A. N. Boyarkina, Izv. Akad. Nauk SSSR, Ser. Fiz. 28, 337 (1964) [Bull. Acad. Sci. USSR, Phys. Ser. 28, 255 (1964)].

${ }^{8}$ F. C. Barker, Nucl. Phys. 83,418 (1966).
}

\author{
${ }^{9}$ N. E. Reid, G. J. Stephenson, Jr., and M. K. Baner- \\ jee, Phys. Rev. C 5 , 287 (1972). \\ ${ }^{10}$ J. M. McKinley, Rev. Mod. Phys. 35, 788 (1963); \\ L. D. Roper, R. M. Wright, and B. T. Feld, Phys. Rev. \\ 138, B190 (1965); J. R. Carter, D. V. Bugg, and A. A. \\ Carter, Nucl. Phys. B58, 378 (1973); D. Dodder, pri- \\ vate communication. \\ ${ }^{11}$ A group at the University of Colorado has done an \\ independent estimate for ${ }^{13} \mathrm{C}$ and obtain results similar \\ to ours. R. E. Anderson, J. J. Kraushaar, E. Rost, \\ and D. A. Sparrow, private communication. \\ ${ }^{12}$ A. I. Yavin, R. A. Hoffswell, L. H. Jones, and T. M. \\ Noweir, Phys. Rev. Lett. 23, 1049 (1966); J. Alster, \\ D. Ashery, A. I. Yavin, J. Duclos, J. Miller, and M. A. \\ Moinester, Phys. Rev. Lett. 28, 313 (1972); J. S. Lilley, \\ D. H. Fitzgerald, C. H. Poppe, S. M. Grimes, and \\ C. Wong, Bull. Am. Phys. Soc. 20, 628 (1975).
}

\title{
Possibility of Detecting Density Isomers in High-Density Nuclear Mach Shock Waves*
}

\author{
Jürgen Hofmann, Horst Stöcker, Ulrich Heinz, Werner Scheid, and Walter Greiner \\ Institut für Theoretische Physik der Universität Frankfurt am Main, Frankfurt am Main, Germany
}

(Received 29 September 1975)

\begin{abstract}
Up to now no experimentally feasible method for detecting abnormal nuclear states has been known. We propose to observe them in high-energy heavy-ion collisions through the disappearance of, or irregularities in, high-density nuclear Mach shock phenomena.
\end{abstract}

Even though nuclear density isomers were suggested recently by several authors,$^{1-5}$ there has been up to now no known experimentally feasible way for their detection. We suggest here a rather simple and unique method for their observation, which is based on high-density nuclear Mach shock (HDNMS) waves and head shock waves occurring during the interpenetration of high-energy heavy ions. ${ }^{6-8}$ Indeed, the recent experiments of Baumgardt et al.$^{8}$ could be consistently interpreted with the shock-wave concept. In particular, these experiments lead to the conclusion that the observed Mach angles cannot be explained with simple sound waves of low amplitude ${ }^{9}$ close to nuclear equilibrium density $\rho_{0}$ but that HDNMS waves are necessary for which the actual density $\rho / \rho_{0}$ is approximately $3-6$. At these densities isomeric or abnormal nuclear states may exist $\mathrm{t}^{1-5}$ which will affect the properties of the nuclear system. The situation can schematically be represented by the compression-energy functional $W_{c}(\rho)[$ Fig. 1(a)]. Its first minimum is associated with the nuclear ground state of binding energy $M_{0} c^{2}-W_{0} \approx 16 \mathrm{MeV}$, and its second minimum, separated by a barrier from the first one, represents the density isomer. This secondary minimum and particularly the binding energy $M_{0} c^{2}-W_{2}$ of abnormal nuclear matter may shift up or down by a few hundred $\mathrm{MeV} .^{1-5}$ Moreover, the compression energy of the isomer, which is determined by the compression constant $K_{2}=9 \rho_{2}{ }^{2}$ $\times d^{2} W / d \rho_{2}^{2}$ of the second minimum, may drastically deviate from that of the ground state $\left(K_{0}\right.$ $\approx 300 \mathrm{MeV})^{8}$ and lead to very high sound velocities in abnormal nuclear matter $c_{s} / c=(\partial p / \partial e)^{1 / 2}$ at constant entropy, where $e=W \rho$ is the energy density and $p=\rho^{2} \partial W / \partial \rho$ is the pressure. Requiring the conservation of flux of baryons, energy, and momentum across the shock front one gets the relativistic Rankine-Hugoniot $(\mathrm{RRH})$ equation

$$
\frac{i_{0}^{2}}{\rho_{0}{ }^{2}}-\frac{i^{2}}{\rho^{2}}+p\left(\frac{i_{0}}{\rho_{0}{ }^{2}}+\frac{i}{\rho^{2}}\right)=0
$$

which uniquely connects the specific enthalpies $i=W \rho+p$ and $i_{0}=W_{0} \rho_{0}\left(p_{0}=0\right)$, the pressure $p$, and the densities $\rho$ and $\rho_{0}$ on the two sides of the shock front. The index zero denotes the unshocked nuclear matter. For the energy per baryon $W(\rho$, $T$ ) in compressed nuclear matter we made the following Ansatz:

$$
W(\rho, T)=M_{0} c^{2}+W_{c}(\rho)+W_{T}(\rho, T),
$$




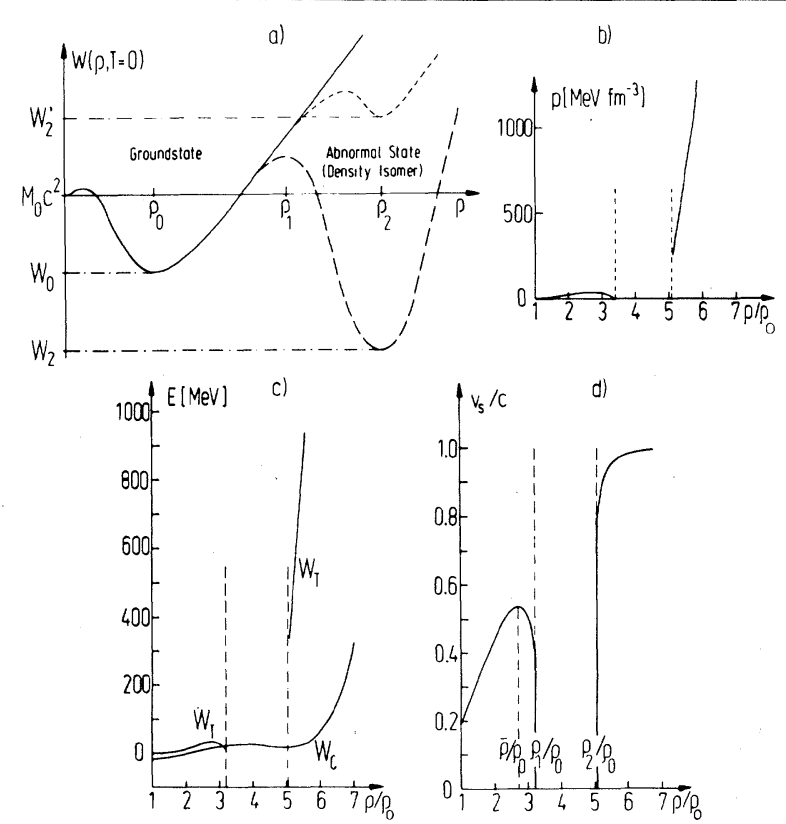

FIG. 1. (a) Various schematic energy functionals $W(\rho, T=0)$. The first minimum at the nuclear groundstate density $\rho_{0}$ and secondary minima of possible density isomers at $\rho_{2}$ are separated by a barrier at $\rho_{1}$. (b) The pressure $p(\rho)$ in the compression region. It vanishes at $\rho_{1}$, becomes negative, and again reaches positive values at $\rho_{2}$. (c) Calculated $W_{c}(\rho)$ and $W_{T}(\rho)$ with $M_{0} c^{2}-W_{0}=-16.456 \mathrm{MeV}$ at $\rho_{0}=0.17 \mathrm{fm}^{-3}, K_{0}=300$ $\mathrm{MeV}$, and $M_{0} c^{2}-W_{2}=15 \mathrm{MeV}$ at $\rho_{2}=5 \rho_{0}$. In the region of negative pressure, i.e., between the maximum of $W_{c}(\rho)$ at $\rho_{1}$ and the secondary minimum at $\rho_{2}$, the $R R H$ equation is no longer valid. The shock phenomena break down, which is indicated by the two dashed lines at $\rho_{1}$ and $\rho_{2}$. (d) The shock velocity $v_{s}(\rho)$ begins at $\rho_{0}$ with the sound velocity $c_{s}=\left(K / 9 M_{0}\right)^{1 / 2}=0.19 c$ and approaches asymptotically $\left(\rho / \rho_{0} \rightarrow \infty\right)$ the velocity of light $c$.

where $M_{0} c^{2} \approx 938.9 \mathrm{MeV}$ is the rest energy per nucleon, $W_{c}(\rho)$ is the above mentioned compression energy per nucleon at zero temperature, for which we use the power expansion

$$
W_{c}(\rho)=A \rho^{5}+B \rho^{4}+C \rho^{3}+D \rho^{2}+E \rho,
$$

and $W_{T}(\rho, T)$ is the thermal energy per nucleon. The coefficients $A-E$ fix the barrier height, isomeric density, and compressibility of the schematic model [Fig. 1(a)]. The pressure $p$ in the compression zone is

$$
p=\left.\rho^{2} \frac{\partial W}{\partial \rho}\right|_{S=\text { const }}=p_{c}+\alpha \rho W_{T},
$$

where $p_{c}=\rho^{2} \partial W_{c} / \partial \rho$ and $\alpha=\frac{2}{3}$ for ideal and Fermi gases. ${ }^{8}$ It is shown in Fig. 1(b). The RRH equa- tion (1) connects the temperature with the density, i.e., $T=T(\rho)$. Hence we obtain within the shock wave $W_{T}(\rho, T(\rho))=W_{T}(\rho)$ from the solution of the quadratic equation

$$
a W_{T}^{2}+b W_{T}+c=0,
$$

where $a=\alpha+1$,

$$
b=\left(W_{0}+W_{c}\right)(\alpha+2)+p_{c} / \rho-\alpha\left(\rho / \rho_{0}\right) W_{0},
$$

and

$$
c=\left(W_{c}+W_{0}\right)^{2}-W_{0}^{2}+W_{0} p_{c}\left(\frac{1}{\rho}-\frac{1}{\rho_{0}}\right)+\frac{W_{c}}{\rho} p_{c},
$$

with $W_{0}=M_{0} c^{2}-16.456 \mathrm{MeV} . W_{T}(\rho)$ is depicted in Fig. 1(c). In the ascending part of the groundstate minimum where the pressure $p$ increases, $W_{T}(\rho)$ behaves as known for normal nuclear matter ${ }^{8}$; i.e., $W_{T}(\rho)$ is a monotonically increasing function of $\rho$, so that the HDNMS wave will propagate through the nucleus and eject the shock particles at the surface. This is also reflected in the behavior of the shock velocity given by

$$
\frac{v_{s}}{c}=\left(\frac{p W(\rho, T) \rho}{\left[W(\rho, T) \rho-W(\rho, 0) \rho_{0}\right]\left[W\left(\rho_{0}, 0\right) \rho_{0}+p\right]}\right)^{1 / 2}
$$

and shown in Fig. 1(d). In the region just mentioned, $v_{s} / c \gg c_{s} / c$ which implies a HDNMS wave, but at a certain density $\bar{\rho}, v_{s} / c$ shows a maximum. As the $W_{c}(\rho)$ barrier maximum is approached the pressure $p$ goes to 0 (because $\partial W /$ $\partial \rho-0)$. Therefore $v_{s}(\rho) / c$ is decreasing, which means that the shock phenomena vanish. Simultaneously $W_{T}(\rho)$ goes to 0 because of the isomeric cooling (the phase transition to the isomer extracts internal energy). At even higher densities $\left(\rho_{1} \leqslant \rho \leqslant \rho_{2}\right)$ the RRH equation is no longer valid.

This behavior indicates the phase transition of nuclear matter. Behind the potential barrier between ground and isomeric states the pressure becomes even negative, which means that the compression zone collapses into a new (abnormal) state which cools the hot ordinary matter. This accelerates the change of nuclear matter. This effect is similar to the condensation of a gas into a fluid (state of higher order) where the van der Waals equation becomes invalid. To stress this point we draw a $p-V$ diagram for $T=0$ and for the variable $T$ in the shocked matter which resembles to some extent that for the van der Waals gas (Fig. 2). For densities higher than the isomeric ground-state density $p_{2}$, the shock phenomenon should develop again. We assume that the strongly compressed head shock moves rather friction-free through the normal nuclear 


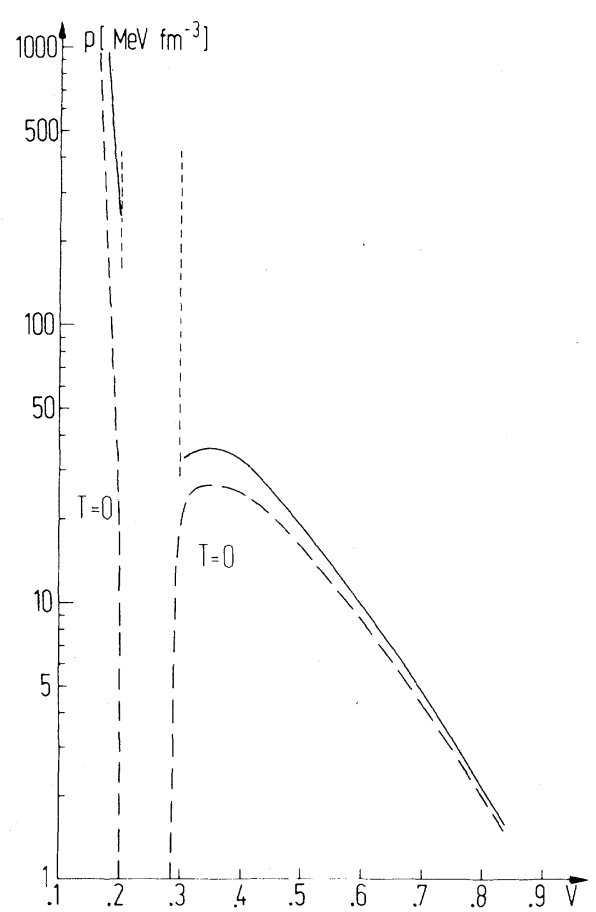

FIG. 2. Pressure-volume $(p-V)$ diagram (for $V=\rho_{0} / \rho$ ) of a qualitative comparison of dense nuclear matter with an isomeric phase. It obviously resembles to some extent that of the van der Waals gas, which also becomes invalid at the condensation point. Dashed curve: isothermal $p-V$ diagram for $W_{T}=0$ (temperature $T=0)$; solid curve: $p-V$ diagram calculated with the temperature dependence $T(\rho)$ obtained by RRH equation.

matter with $v_{\text {HS }} \approx v_{\text {ion }}$. This kind of superfluidity can happen via pion condensation, for which $\pi^{+}$$\pi^{-}$layers with-depending on the proton-neutron ratio-autoionizing $\pi^{-}$can occur. The $\pi^{+}$layer is tightly bound to the highly compressed core of the projectile, which so stabilizes itself. We then expect, because of the reaction $\pi^{+}+n \rightarrow p$, proton-rich (neutron-poor) nuclei to dominate the fragmentation products and an excess of free $\pi^{-}$ over $\pi^{+}$.

Now, from the measurement of the Mach angle $\varphi$ one can derive the Mach shock velocity, $v_{\mathrm{MS}}$, via the relation ${ }^{8}$

$$
\cos \varphi=v_{\mathrm{MS}} / v_{\text {ion }} \text {. }
$$

For ordinary nuclear matter one can expect qualitatively the following results: At low projectile velocities (energies) the Mach angle will be small and increase with projectile energy. It will not tend to $90^{\circ}$ with asymptotically high projectile energies, as one would expect according to nonrelativistic gas dynamics, but should have a maxi-

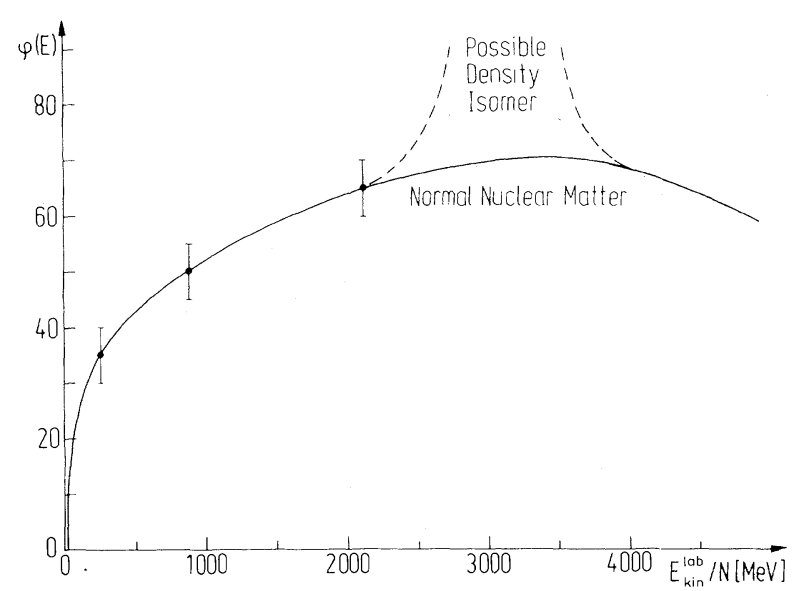

FIG. 3. The Mach angle $\varphi(E)$ as a function of the projectile energy (per nucleon, $N$ ). The presently available experimental results (Ref. 8) are indicated. The consequence of a possible density isomer will affect the Mach angles as schematically indicated by the dashed curve.

mum value remarkably smaller than $\arccos \left(c_{s}\right)$ $c) \approx 80^{\circ}$, because of the velocity of light as an upper limit for $v_{\text {ion }}$. $\varphi$ will again tend to zero at very high projectile energies $\left(v_{\text {ion }} / c \approx 1\right)$ because $v_{\mathrm{MS}} / c$ is a monotically increasing function of $\rho$ and also tends to 1 for $\rho \gg 1$ [Fig. 1(d)].

If a density isomer exists, this behavior will be drastically changed: The Mach shock velocity decreases to zero for a certain density region [Fig. 1(d)], so that the observable Mach angle will reach $90^{\circ}$. Then the Mach angle should abruptly vanish in the energy interval where $\rho_{1}$ $\leqslant \rho \leqslant \rho_{2}$ and suddenly for $\rho>\rho_{2}$ reappear again at $90^{\circ}$ to follow the above mentioned trend to zero degrees (Fig. 3). This can serve as a unique signature for density isomers or abnormal behavior of nuclear matter. It is interesting to notice that the presently available experimental data ${ }^{10}$ show a decrease of the Mach shock velocity from 0.87 to $2.1 \mathrm{GeV} /$ nucleon with a maximim at 0.87 $\mathrm{GeV} /$ nucleon; see the following table:

\begin{tabular}{cccc}
$\begin{array}{c}E_{\text {kin }} \text { lab } \\
(\mathrm{GeV} / \text { nucleon })\end{array}$ & $\frac{v_{\text {ion }}}{c}$ & $\begin{array}{c}\varphi_{\text {exp }} \\
(\mathrm{deg})\end{array}$ & $\frac{v_{\mathrm{MS}}}{c}=\frac{v_{\text {ion }}}{c} \cos \varphi_{\text {exp }}$ \\
\hline 0.25 & 0.61 & 35 & 0.50 \\
0.87 & 0.87 & 50 & 0.56 \\
2.1 & 0.95 & 65 & 0.40
\end{tabular}

This is perhaps a first indication for some special structure of $W(\rho, T)$, i.e., abnormal behavior of nuclear matter. Nevertheless to definitely prove or disprove the existence of density isomers we suggest repeating the HDNMS-wave ex- 
periment by bombarding, e.g., ${ }^{107} \mathrm{Ag}$ or ${ }^{208} \mathrm{~Pb}$ with ${ }^{16} \mathrm{O}$ in small steps of incident energy (about 50 $\mathrm{MeV} /$ nucleon) from 0.05 to $2.1 \mathrm{GeV} /$ nucleon (if possible higher) and systematically measuring the shock angles. ${ }^{12}$ These measurements will enable us to explore the energy function $W(\rho, T)$, its eventual isomer structures, and also the temperature behavior of a highly compressed nuclear gas. Such experiments should presently be possible without major difficulties.

We thank Professor E. Schopper for many valuable discussions.

*Work supported by Bundesministerium für Forschung und Technologie and by Gesellschaft für Schwerionenfor schung.

${ }^{1}$ A. R. Bodmer, Phys. Rev. D $\underline{4}, 1601$ (1971).

${ }^{2}$ T. D. Lee and G. C. Wick, Phys. Rev. D 9,2291 (1974).

${ }^{3}$ T. D. Lee, Rev. Mod. Phys. 47, 267 (1975).

${ }^{4}$ T. D. Lee and M. Margulies, Phys. Rev. D 11,1591 (1975).
${ }^{5}$ A. K. Kerman, in Proceedings of the Second High Energy Heavy Ion Summer Study, Berkeley, California, July 1974, edited by L. Schroeder, Lawrence Berkeley Laboratory Report No. LBL 3675 (unpublished).

${ }^{6}$ W. Scheid, H. Müller, and W. Greiner, Phys. Rev. Lett. 32, 741 (1974).

'J. Hofmann, W. Scheid, and W. Greiner, "Thermal Excitation of Nucleons in Nuclear Shock Waves" (to be published).

${ }^{8}$ H. G. Baumgardt, J. U. Schott, Y. Sakamoto, E. Schopper, H. Stöcker, J. Hofmann, W. Scheid, and W. Greiner, Z. Phys. A 273, 359 (1975).

${ }^{9}$ A. E. Glassgold, W. Heckrotte, and K. M. Watson, Ann. Phys. (N.Y.) 6,1 (1959).

${ }^{10} \mathrm{~A}$ recalibration of the data of Ref. 8 has shifted the experimental Mach angles to somewhat larger values as shown in Table I (E. Schopper, private communication).

${ }^{11}$ The occurrence of transparency-as postulated by M. I. Sobel, P. J. Siemens, J. P. Bondorf, and H. A. Bethe, to be published-can presently not be ruled out.

${ }^{12}$ The strength of the density isomer may depend on the number of nucleons. This can be experimentally found out by systematically choosing heavier projectile and target nuclei and repeating the excitation function of the Mach angle.

\title{
Homogeneous Broadening of Optical Transitions in Organic Mixed Crystals
}

\author{
Harmen de Vries and Douwe A. Wiersma \\ Laboratory for Physical Chemistry, University of Groningen, Zernikelaan, Paddepoel, Groningen, The Netherlands
}

(Received 21 October 1975)

\begin{abstract}
We have used the phenomenon of laser-induced molecular photodissociation to determine the homogeneous linewidth at $2 \mathrm{~K}$ of the origin (zero-phonon line) and a vibronic transition in the mixed-crystal absorption spectrum of dimethyl $s$-tetrazine in durene. From the measured 55- $\mathrm{MHz}$ (upper limit) homogeneous width of the origin we conclude that in the vibrationless excited state coherence persists at least during the 6-nsec lifetime. The 29$\mathrm{GHz}$ homogeneous vibronic linewidth is ascribed to vibrational relaxation.
\end{abstract}

It is still mainly a matter of speculation as to what extent the linewidths in the low-temperature "sharp" line absorption and emission spectra of organic mixed crystals are inhomogeneously broadened. ${ }^{1}$ In organic crystals the only report of a homogeneous linewidth of a vibrationless transition comes from Hochstrasser and $\mathrm{Li}^{2}$ who showed that the origin (zero-phonon line) of the lowest singlet state in azulene as guest in naphthalene was mainly homogeneously broadened. In this exceptional case there is an extremely fast (2.6-psec) non radiative electronic relaxation process that is responsible for the observed 2$\mathrm{cm}^{-1}$ origin linewidth. Recently Marchetti, McColgin, and Eberly ${ }^{3}$ reported a measurement of the homogeneous linewidth of a vibronic transition in the spectrum of pentacene embedded in a $p$-terphenyl host crystal. The underlying homogeneous linewidth of the $3-\mathrm{cm}^{-1}$-wide vibronic transition was found to be $0.026 \mathrm{~cm}^{-1}$ and the authors concluded from this that vibrational relaxation in this state was as slow as 200 psec. The question of course arises whether such a slow vibrational relaxation process is typical for organics at low temperature or that again an exceptional case was studied. We have designed an optical "hole-burning" experiment that not only answers this latter question, but also permits us, for the first time, to measure directly the underlying homogeneous linewidth of a zero-phonon 